\title{
Wireless Smell System for Hazardous Gases Detection
}

\section{Pablo Gómez ${ }^{1}$ Cristhian Duran ${ }^{1}$ and Rafael Acosta ${ }^{2}$}

1 Multisensor System and Pattern Recognition Research Group, Electronic Engineering Program, Engineering and Architecture Faculty, Universidad de Pamplona, Km 1 Vía Bucaramanga, Pamplona (N.S.), Colombia.

2 CEDRUM NDS Research Group, Servicio Nacional de Aprendizaje (SENA), Cúcuta (N.S.), Colombia

* Correspondence: ingpablogomez@gmail.com; cmduran@unipamplona.edu.co Tel.: +57-311-5418608

\begin{abstract}
This study describes the design and implementation of an electronic nose, which was applied to classify and identify hazardous gases generated in underground coal mines. For this purpose, an electrochemical sensor array was used to detect a set of toxic gases. This work illustrates the electronic components of a wireless multisensory system for the toxic gases detection on indoor environments (i.e., underground mines), which was performed with reduced size, low cost and low electrical consumption, in order to detect different compounds using the basic principle of operation of each component to be applied to the target gas. Furthermore, the sample collection, data communication and data processing in real time obtained an excellent performance for gas sensing and even to measure the concentration level of the chemical volatile compounds transmitted from different points of the detection zone.

The results demonstrated that using a wireless electronic nose for toxic gases detection was possible to reach a success rate of discrimination of $97 \%$, using principal components analysis (PCA) and Linear Discriminant Analysis (LDA).
\end{abstract}

Keywords: electronic nose; wireless system; gas sensors; coal mines; processing; wireless

\section{Introduction}

Nowadays the Electronic Nose, is defined as a measurement instrument that comprises of a set of chemical gas sensors and pattern recognition methods able to recognize simples and complex odors [1], which have had a great applicability in the food industry production (i.e., wine ageing detection, evaluation of peach quality, prediction of acidity, soluble solids and firmness of pear, evaluation of tomato quality, etc.) [2-8]. Also, there are several industrial sectors which have high risks of explosions into different environments $[9,10,11,12]$. The explosions are a phenomenon that take place in an area due to overpressure in a short space of time, with eventually cause human losses and the destruction of the entire or partial of the facility.

Underground coal mines generate a highly toxic and explosive enviroments due to the great amount of gases which are absorbed by the coal $[9,10,11]$. All compounds belong to the methane principally, one of the most dangerous gas is the "gray gas", which consists of methane gas created by the decomposition of organic materials within the coal itself [19]. The gases generated inside of coal mines are such as: Carbon monoxide, carbon dioxide, methane, hydrogen sulfide and in some cases depending on the type of coal since it can find even of ethane in big quantities $[14,15]$. Besides, the toxicity and the possibility to burs these gases present is that makes the work of the miners highly dangerous. With the presence of toxic gases, the coal mines must be permanently ventilated to prevent the concentrations of the dangerous volatile substances avoiding poisonings and/or explosions [13]. It is clear than human nose is not trustworthy as a toxic detection system to alert of the presence of toxic gas like the methane due to it is odorless and colorless. On the other hand, this 
gas emitted in any place (like coal mines) in combination with oxygen can be highly explosive and poisonous.

Therefore, a reliable equipment used as an Electronic Nose would contribute to the safety in coal mines $[9,11]$, since it has the capacity to detect a set of volatile substances and allows that these devices have a big applicability in the industrial sector. The electronic nose can identify the leak of a certain gas or emissions of a dangerous mixture; with this information, emergency methods can select the most effective protection and containment strategy for each specific case. If this technology is related to a wireless data transmission, which has had a rapid development in recent years in different sectors (security, environment, industry, agriculture, etc.) [7,14-17], a Wireless Electronic Nose system would have great advantages, in addition to a greater reliability to obtain a good data set; the most recognized advantage is the low cost of the installation due to a large sections of certified wiring, and are not required under hostile circumstances, achieving a reliable and low-cost equipment for coal mines [18].

\section{Materials and Methods}

\subsection{Electronic Nose}

It is important to consider fundamentally the concept and use of an Electronic Nose (E-Nose) that comprises of a measuring chamber with a gas sensors array coupled to two vacuum pumps, where one of them is used to supply the sample of toxic gases and another pump to wipe the measuring chamber.

This prototype or measurement system consists of two main stages: The first stage contains the sampling system, the second stage is the data measurement and information processing that includes the computer which processes the information from an acquisition board (e.g, wireless card).

At the beginning of the process, a zone of the coal mine releases a toxic gas sample which it is trapped and conditioned by means of a sampling stage, afterwards a sensor array acquired the data when they are pre-heating to get the optimal operational values, once the sensors are triggered, a vacuum pump is activated to purge the sensor chamber to eliminate traces of the previously acquired sample and in this way avoiding to contaminate the next sample. Figure 1 depicts a scheme of the sensor sensing

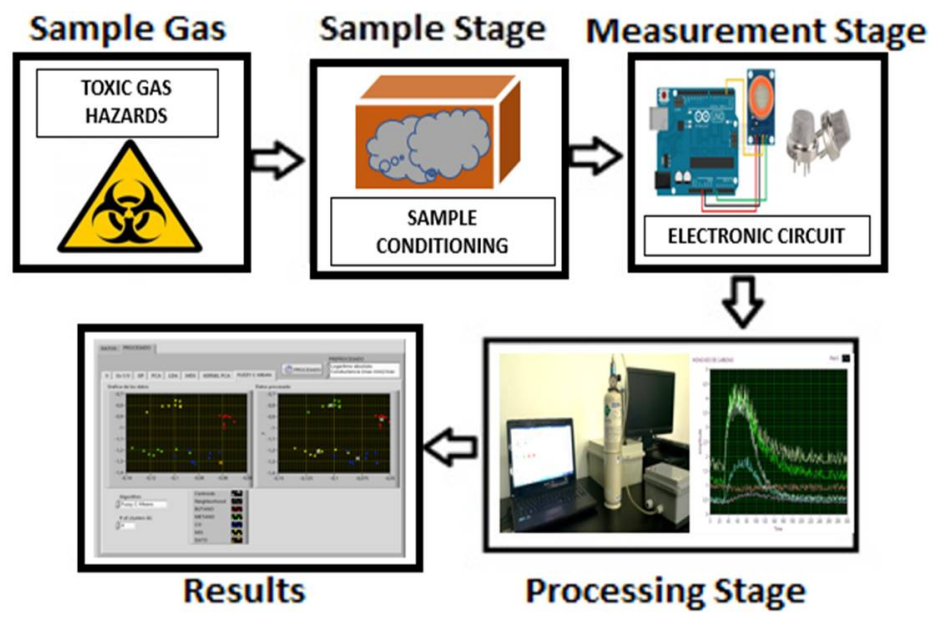

Figure 1. Stages of an Electronic Nose

To acquire the information from the measurement chamber, another pump is activated to send the gas generated in the area where a node (i.e., point of intersection/connection within a network), which is located inside the mine, the gases are measured through the measurement chamber. The pump is turned-off and the purge pump is activated for recovery time of the sensors. Thus, the 
sensors return to a normal operation values and eliminate the saturation present by the reaction generated by the sample acquired previously.

\subsection{E-Nose components}

The following section shows the electronic components used to develop the wireless E-Nose Network (WENN) system.

\subsubsection{Measurement Chamber:}

The measurement chamber was manufactured in an airtight acrylic container (See Figure 2), which houses the sensor array. In this chamber the information is acquired and then sent to the acquisition board and the data processing stage. The dimension of the sensor chamber was $6.5 \mathrm{~cm} x$ $6.8 \mathrm{~cm} \times 4.1 \mathrm{~cm}$ respectively, with a total volume of $181.22 \mathrm{~cm}^{3}$. Figure 2 shows the measuring chamber design with all sensors located in the bottom part of the chamber and the connections of the vacuum pumps.

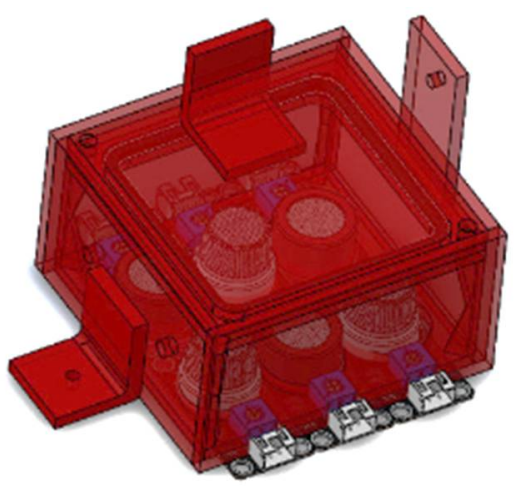

Figure 2. Measurement Chamber.

The measuring chamber has two holes in one side, which were made for inlet and outlet couplings for the gases. This chamber has a cover which can be removed in order to carry all samples towards the sensors.

\section{0}

\subsection{Vacuum Pump:}

One of the most critical part of the wireless electronic nose nodes is the way in which the sample is loaded and unloaded in the measurement chamber, since in the environment in which it is going to be found (i.e., a mine of coal), the traditional methods to reset the sensors are not appropriate [20]. Before of the real tests and samples collection, the sensor chamber had to be open because it needed to bring environmental air to achieve the recovery time of the sensors. The next stage was to use two vacuum pumps referenced as T2-03 series diaphragm made by Parker Brand Mark [21], where one of them carry the sample and the second one extracts all gases present inside the measurement chamber, achieving a recovery in a short time (see Figure 3). The vacuum pumps were connected to a power supply of $5 \mathrm{VDC}$, with a low consumption of 2.3 Watts to $386 \mathrm{~mA}$. The pumps were used in On/Off mode, since the injection and purge of the sample towards the measuring chamber was made with a constant air flow. The maximum flow was set in $2.5 \mathrm{~L} / \mathrm{min}$ with a maximum pressure of 12 PSI, according to the technical sheets supplied by the manufacturer. This kind of vacuum pump can be used in portable applications and it was appropriate to perform the electronic nose. 
115

116

117

118

119

120

121

122

123
124

125

126

127

128

129

130
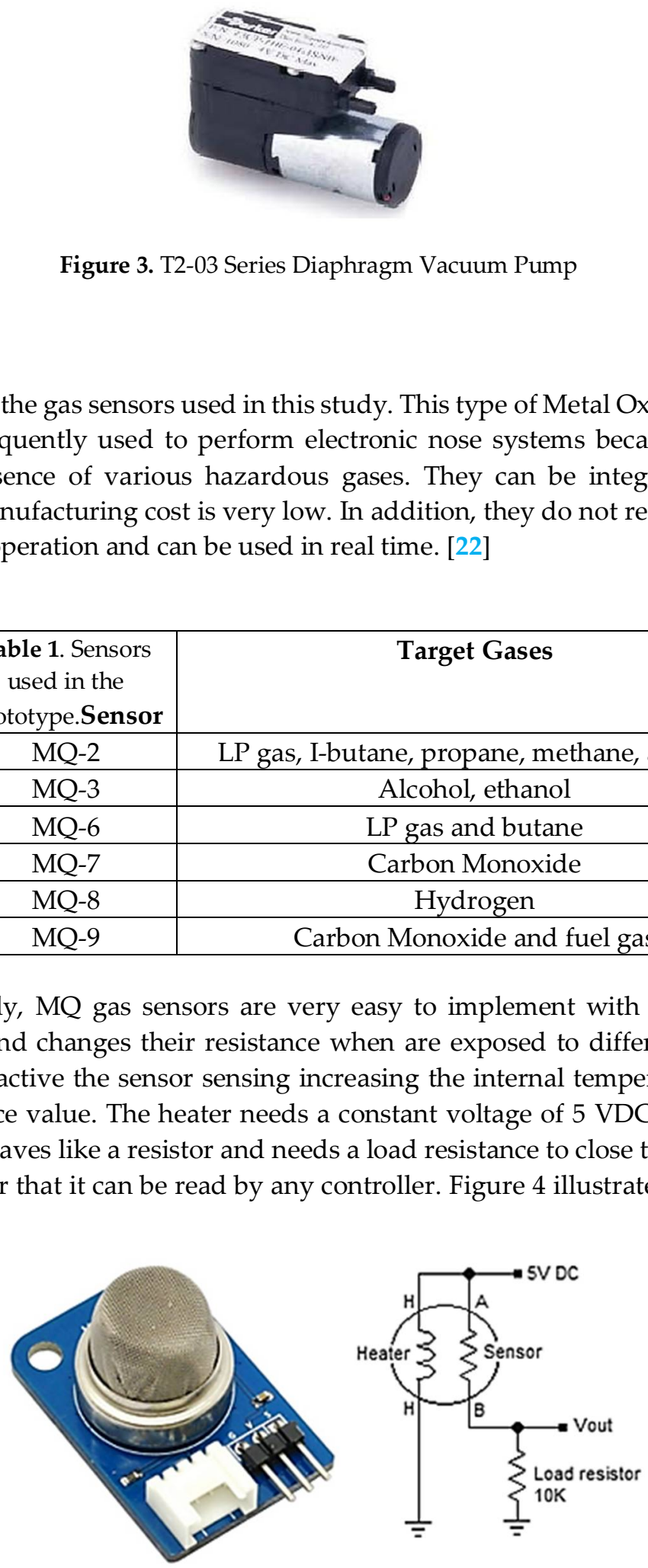

Figure 3. T2-03 Series Diaphragm Vacuum Pump

\subsection{MQ Gas Sensors:}

Table 1 illustrates the gas sensors used in this study. This type of Metal Oxide Gas Sensor (MOS) is one of the most frequently used to perform electronic nose systems because they have a high sensitivity in the presence of various hazardous gases. They can be integrated into a portable equipment and the manufacturing cost is very low. In addition, they do not require instrumentation too complex for their operation and can be used in real time. [22]

\begin{tabular}{|c|c|}
\hline $\begin{array}{c}\text { Table 1. Sensors } \\
\text { used in the } \\
\text { Prototype.Sensor }\end{array}$ & Target Gases \\
\hline MQ-2 & LP gas, I-butane, propane, methane, alcohol \\
\hline MQ-3 & Alcohol, ethanol \\
\hline MQ-6 & LP gas and butane \\
\hline MQ-7 & Carbon Monoxide \\
\hline MQ-8 & Hydrogen \\
\hline MQ-9 & Carbon Monoxide and fuel gas \\
\hline
\end{tabular}

As said previously, MQ gas sensors are very easy to implement with any controller. These sensors are resistive and changes their resistance when are exposed to different gases. Thus, they have a heater wire to active the sensor sensing increasing the internal temperature which causes a change in the resistance value. The heater needs a constant voltage of 5 VDC for proper operation and the gas sensor behaves like a resistor and needs a load resistance to close the circuit and thereby apply a voltage divider that it can be read by any controller. Figure 4 illustrates the electrical circuit of gas sensors [22,25].

Figure 4. Electrical circuit of a gas sensor

As soon as the module is powered, these sensors started to warm up, in this time it was necessary to wait a few minutes to obtain a satisfactory sensing a because the implemented application need concentration values of the gases which were necessary to calibrate the sensor using known concentrations. 
138

139

140

141

142

143

144

145

146

147

148

149

150

151

152

153

154

155

156

157

158

159

160

161

\subsection{Sensor Array:}

Figure 5 depicts the sensor chamber where were placed all sensors required in this study. A circuit board made by company Dfrobot was implemented for sensor conditioning where each of them enabled the mounting inside the measurement chamber. Figure 5 shows the circuits placed along the lower part of the box.

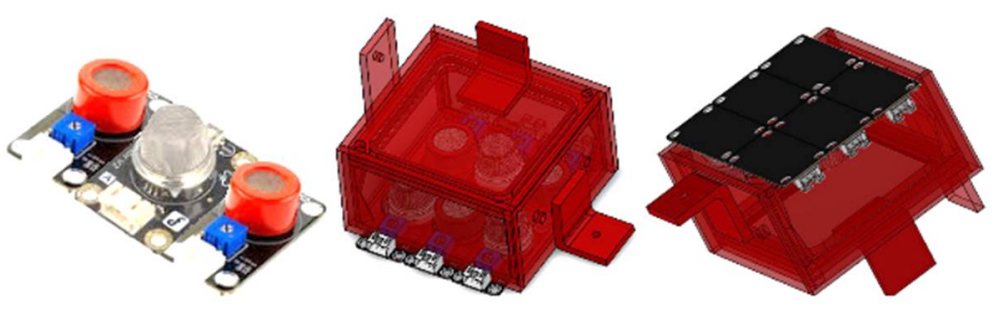

Figure 5. Gas sensors located into the measurement chamber.

\subsection{Power and acquisition Board:}

The main function of the power board was supply voltage (heater resistor and sensor) for all gas sensors, an ARDUINO-UNO board for data acquisition [26] (see Figure 6) and the vacuum pumps for flow control. This board was performed for sensors conditioning, to activate the pumps through of digital outputs and send the sensor responses to the ARDUINO board. The power supply has the enough energy to activate the components.
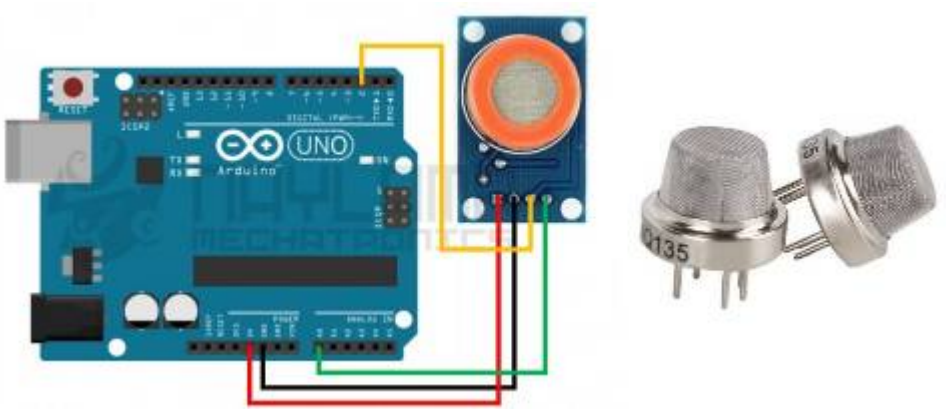

Figure 6. Implementation of MQ sensors with an arduino board

The board has a set of variable outputs for the adaptation of the sensor signals in order to use other development boards that do not works with voltage levels from 0 to 5VDC. The main components are shown below:

- MOSFET IRFZ44N: This electronic device was used for activating the pumps and sensors since they work as a relay (operates with currents up to $50 \mathrm{Amps}$ ). The MOSFET was selected because the response time it is fast and stable. It can also use to vary the voltage applied to the pumps via PWM, whether it is required. 
Table 2. MOSFET IRFZ44N transistor

\begin{tabular}{|c|c|}
\hline Parameter & Value \\
\hline Drain-Source Voltage (VDSS) & $55 \mathrm{~V}$ \\
\hline Drain Current-Continuous (ID) & $49 \mathrm{~A}$ \\
\hline Total Dissipation (PD) & $94 \mathrm{~W}$ \\
\hline
\end{tabular}

163

164

165

166

167

168

169

170

171

In this study a general purpose optocoupler with reference 4N25 was used, due it was required to isolate the control signal from the board and the power stage for the activation of the sensors and pumps.

\subsection{Switched-mode power supply:}

Figure 7 depicts the power supply which it can convert an input voltage up to a maximum of 15 VDC, appropriate for this application. It was selected as a stable and reliable source, because it was needed to reduce the voltage from 12VDC to 5 VDC and with it guarantees a maximum output of 3 Amps.

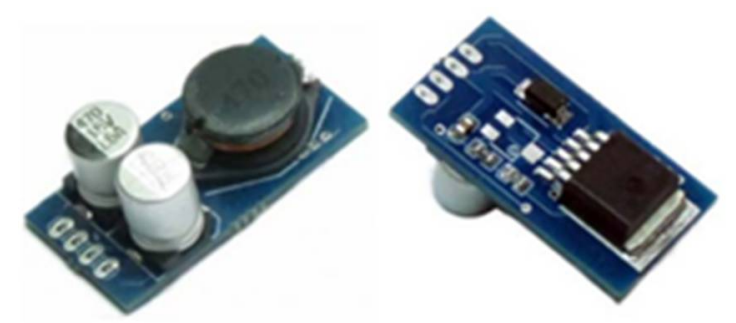

Figure 7. Power supply based on the integrated LM2576

\subsection{Activation Electronic circuit:}

Figure 8 shows the circuit used for pump activation. It can be seen that voltage of 5 VDC is applied to the input, which it is activated with the digital output of the Arduino board. A resistance of $100 \mathrm{Ohm}$ was used since it is necessary to feed the Gate pin of the Mosfet. The circuit can be used with higher voltages, as shown in the Figure 8. Three similar circuits were used to control the sensor array and pumps. Furthermore, the isolation of the control stage (left-side) and the power-stage (right-side) was necessary to prevent against countercurrents since it can affect the performance of the board.

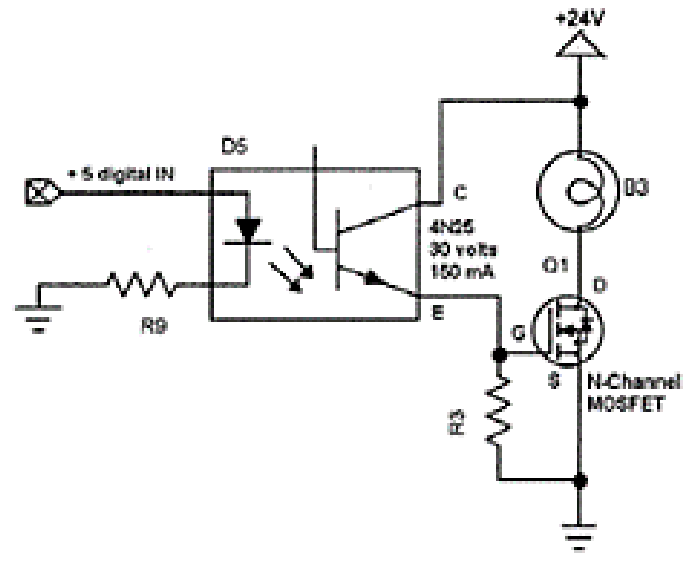

Figure 8. Activation circuit.

The design was made by Eagle 6.3.0 software (see Figure 9). 
186

187

188

189

190

191

192

193

194

195

196

197

198

199

200

201

202

203

204

205

206

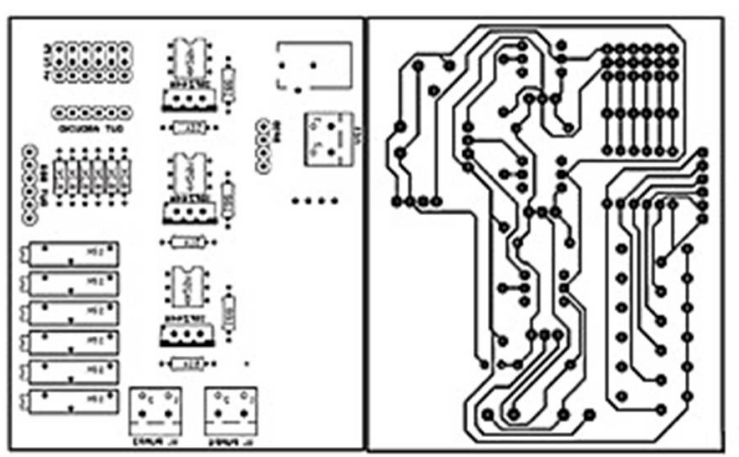

(a)

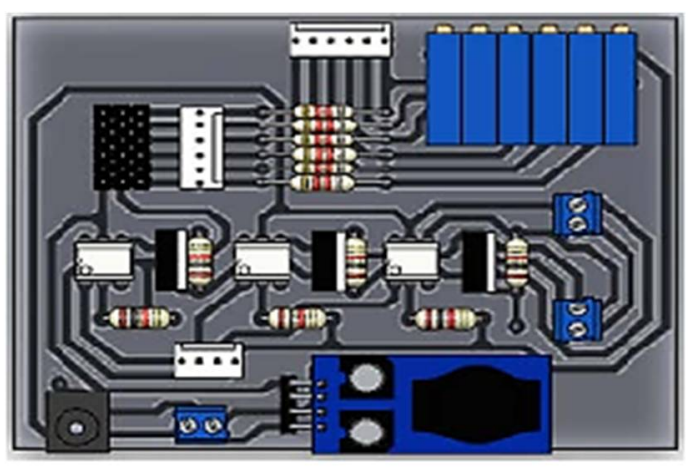

(b)

Figure 9. (a) PCB layout and b) Power board components

Table 3 illustrates the overall characteristics of the board.

Table 3. Power Board Characteristics

\begin{tabular}{|c|c|}
\hline Parameter & Characteristics \\
\hline Control Inputs & $\begin{array}{c}\text { Three control bits from Arduino } \\
\text { One common ground with BeagleBoneBlack Board }\end{array}$ \\
\hline Analog Inputs & Matrix of 6x3 male pins for sensor location \\
\hline Vacuum Pump Control & Two 12V Output, PWM Enable \\
\hline Input DC Voltage & One 12V Input \\
\hline Output to Sensors & Six 5V Outputs to Android Board \\
& Six 5V Outputs to BeagleBoneBlack Board \\
\hline Output DC Voltage & One 12V Outputs \\
\hline
\end{tabular}

\subsection{Power Supply:}

A power supply of 12 VDC and 11 Amps manufactured by Mean Well [27] was used to feed the board, the vacuum pumps and sensor array. This type of source was selected because it has the necessary specifications for the proper functioning of the sensors and the development board. Besides, it provides an IP 65 safety standard suitable to be applied in coal mines (see Figure 10).

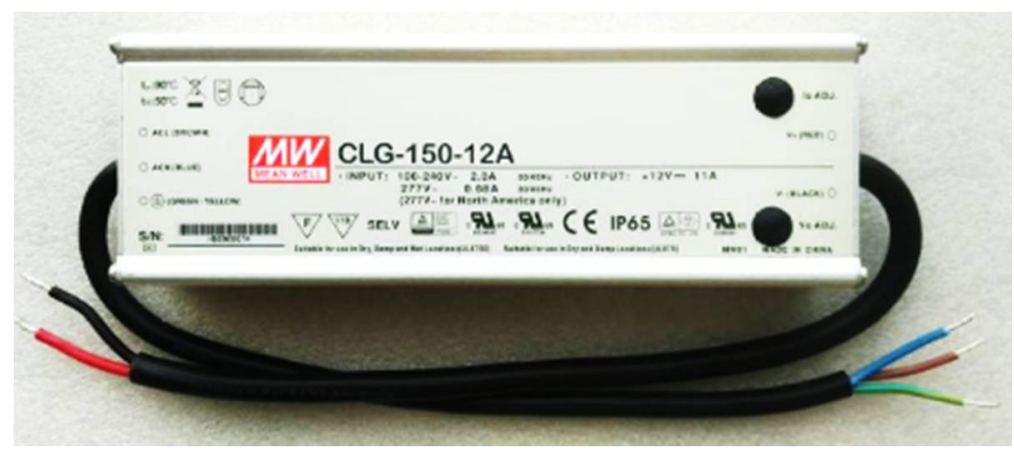

Figure 10. Mean Well Power Supply. 


\subsection{Transmission and reception modules Xbee PRO ZB (S2B):}

The Radio Frequency (RF) module used in this application was a Xbee PRO ZB (S2B) device, manufactured by Digi Electronics (Digi International, 2015), which works under the Zigbee protocol (IEEE 802.15.4). The Xbee Pro modules were chosen due to their wide transmission range (i.e., use of free radio bands), low cost and easy programming since they allow an Application Programming Interface (API) Mode; this configuration can sends and receives information in the form of data packets whereas include additional data. These modules were necessary for the operation of the protocol because are included with the data in each packet.

The Figure 11 shows the series 2 module with a microchip device manufactured by Ember Networks, used for the implementation of a type mesh network which is goods of Zigbee Alliance. With this type of mesh network, it is possible to create a wireless sensor networks with a robust assembly that can generate a large amount of data set to support complex human interactions. By means of the wireless modules, several complex network type mesh can be created, allowing access to remote points using intermediate nodes such as routers. These types of modules automatically generate the network without human intervention and have the ability to configure themselves when a node fails. In addition, the network can solve the best route for a given package. [24].

Figure 11. Xbee Pro ZB Series 2 Module.

230 Table 4 illustrates the most important characteristics of the Xbee PRO modules. 
Table 4. Xbee PRO Characteristics.

\begin{tabular}{|c|c|}
\hline \multicolumn{2}{|c|}{ Specifications Xbee PRO ZB Series 2} \\
\hline \multicolumn{2}{|c|}{ Performance } \\
\hline Data Velocity & RF $250 \mathrm{Kbps}$ \\
\hline Indoor / Urban range & Up to $90 \mathrm{~m}$ \\
\hline Outdoor line-of-sight range & Up to $3200 \mathrm{~m}$ \\
\hline Transmit Power Output & $63 \mathrm{~mW}(+18 \mathrm{dBm})$ \\
\hline Receiver Sensitivity & 102 dBm (1\% Packet Error Rate) \\
\hline \multicolumn{2}{|c|}{ Power Requirements } \\
\hline Supply Voltage & $2,7-3,6 \mathrm{VDC}$ \\
\hline Operating Current (Transmit) & $205 \mathrm{~mA}$ \\
\hline Operating Current (Receive) & $47 \mathrm{~mA}$ \\
\hline Power-down Current & $3,5 \mathrm{~mA} @ 25^{\circ} \mathrm{C}$ \\
\hline \multicolumn{2}{|c|}{ General } \\
\hline Interference Immunity & DSSS (Direct Sequence Spread Spectrum) \\
\hline Operating frecuency band & ISM $2.4 \mathrm{GHz}$ \\
\hline Number of Channels & 15 Channels \\
\hline Operating temperature & $0^{\circ} \mathrm{C}$ to $85^{\circ} \mathrm{C}$ (Industrial) \\
\hline
\end{tabular}

\subsection{Data acquisition card:}

The selected card was an Arduino UNO, which has the concept of free software and free hardware and it is compatible with communication modules on the 802.15.4 platform, thus it is suitable to use with Wireless sensor networks (WSN) prototypes. In addition to the above, an outstanding feature is the great variety of boards to add additional functions that these electronic boards account for.

The board used is called "Xbee Shield", being used to perform wireless communications under the ZigBee protocol, another point to consider is that in order to carry out the programming and communication of the Xbee, the Xbee explorer module was used. This module carry out the programming and communication of the Xbee, which has a direct USB connection to the PC.

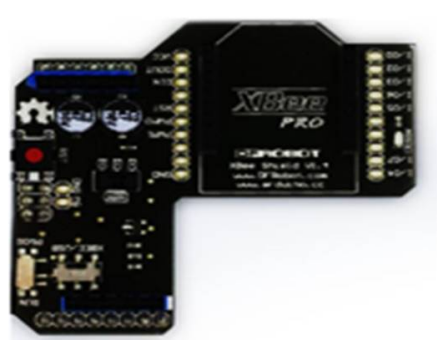

(a)

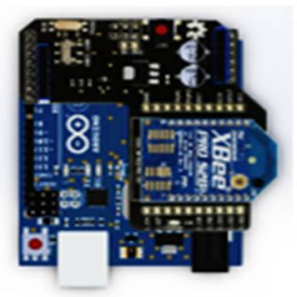

(b)

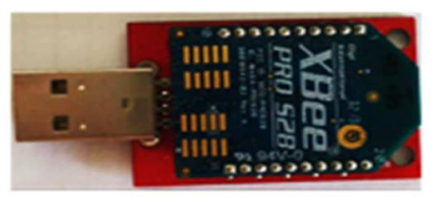

(c)

Figure 12. (a) Xbee Shield, (b) Xbee Shield coupled to Arduino UNO (c) and Xbee Explorer. 
In order to avoid damage of components of the electronic nose, an inert plastic and robust box was used with IRAM (Instituto Argentino de Normalización y Certificación), according to the international standard IEC 670: $1989+$ A1: 1994. [29].

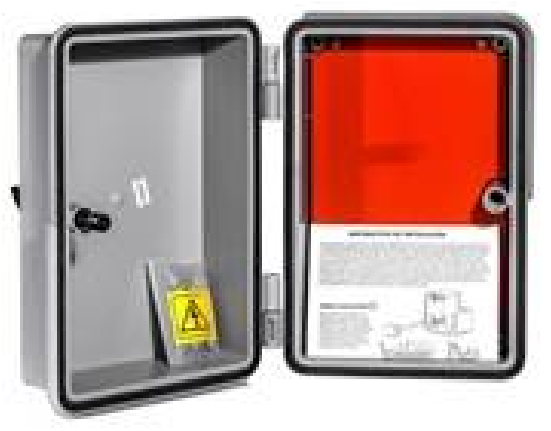

Figure 13. Inert plastic box

\section{Results and discussion:}

Figure 13 illustrates an excellent classification applying PCA, where the discrimination of clusters can be observed directly and with suitably spaced between categories. To perform the data pre-processing, the following statistical parameter was used: $\Delta G=G_{\max }-G_{\min }$, where $G_{\max }$ it is the maximum response of the sensor and $G$ min correspond to the minimum value (amplitude) of the sensor response. A success rate of $97 \%$ of discrimination was obtained choosing only 2 PC's.

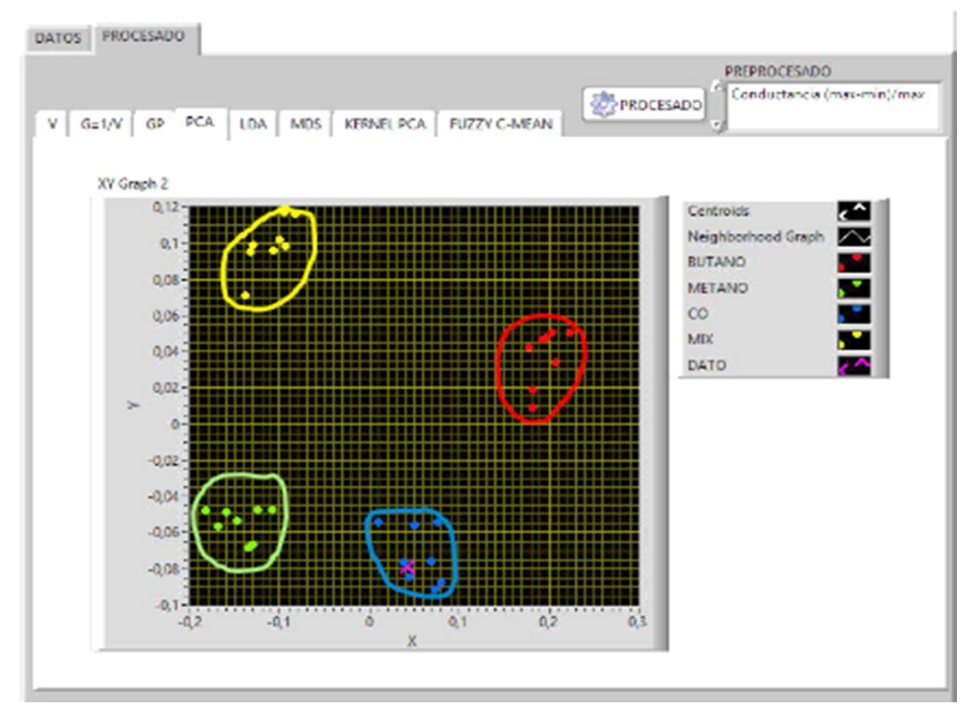

Figure 13. PCA with (Gmax-Gmin)/Gmax

On the other hand, the results applying LDA were excellent, obtaining a success rate in the discrimination of the measures was $97 \%$ using 2 canonical variables. To perform the data preprocessing the same statistical parameter used previously was applying to the data set: $\Delta \mathrm{G}=\mathrm{G}_{\max }-$ Gmin. 
Figure 14. LDA with (Gmax-Gmin)/Gmax

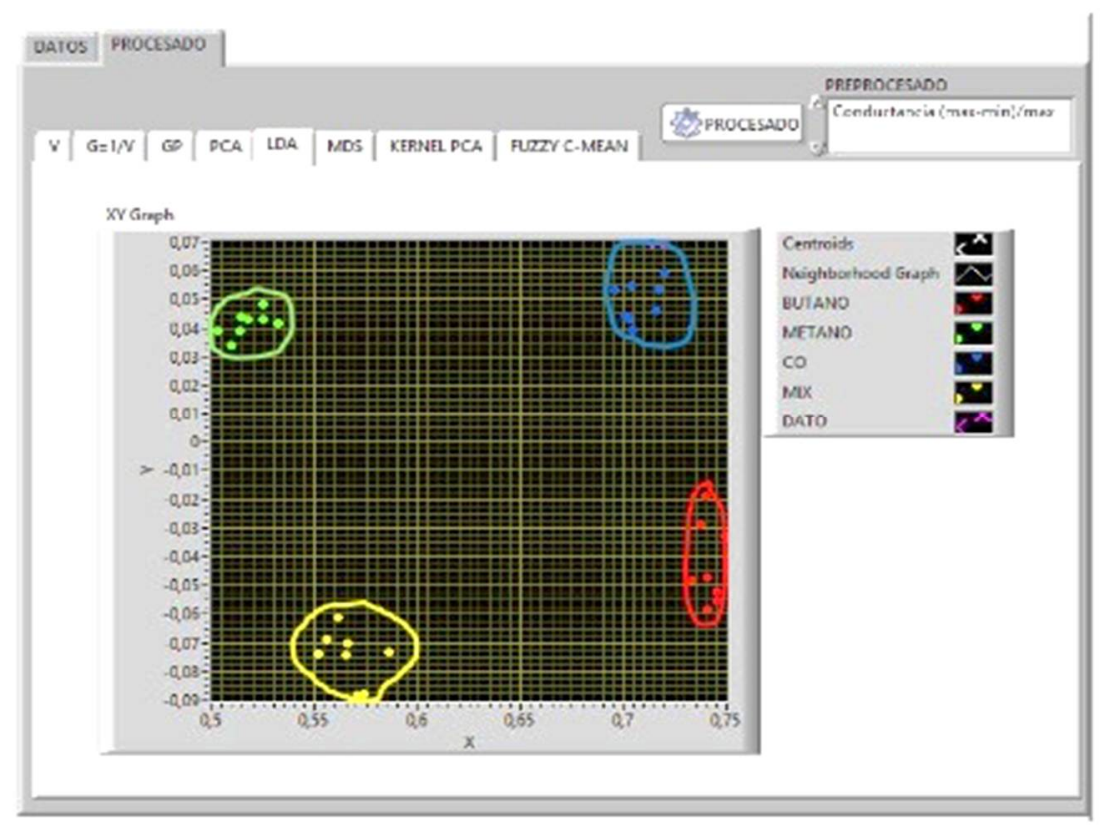

The results showed in this study demonstrates that it is very important and necessary to find the type of electrochemical gas sensors for each application, depending of the characteristics and variety of sensors on the market, since with this preliminary study it was possible to select the most suitable of them to achieve results. Two references were taken into account for the analysis of hydrogen using the Figaro TGS821 sensor [23] and MQ-8 Sensor [22]. Within the characteristics linked to the project, such as the response time where the Figaro sensor has a greater range of sensitivity since it works from a range of $10 \mathrm{ppm}$ while the MQ Sensor reach $100 \mathrm{ppm}$, and a second characteristic to analyze is the amount of volatiles with which they responded, being the MQ-8 sensor the one that reacts to a greater number of volatiles. It was noted that MQ-8 sensor had a wide difference with the Figaro sensor. For all the above, the MQ gas sensor series was used for the prototype, due to its low cost, versatility and wide range of volatile detection, helping to identify the gases emitted inside a coal mine.

For the data transmission stage, where three Wifi, Bluetooth and Zigbee communication protocols were analyzed to find of the most suitable to be applied in this project. The main factor to select the standard communication protocol was found through the mesh or tree-like type topology, average cost, minimum transmission and a distance of 60 meters and transfer rate higher than 40 Kbps. By analyzing the three protocols, only the Zigbee protocol was appropriate [10, 18], since it had each one of the factors and also a security system for the data transmission that are a key point of the technology. In this case it uses the IEEE 802.15.4 MAC security protocol, which specifies four security services such as: 1) Access control: It means that the devices maintain a list of the devices checked for the network. 2) For encrypted data, where the data uses an encryption with a 128 bit code. 3) Integration of frames, protect the data from being modified by others and 4) Sequences of refresh, check that the frames have not been replaced by others, this happens because the network controller checks these refresh frames and their value to see if they are the expected ones. In stage 1, where the sensors must be re-established to make a new sample collection. The accurate times must be set for activation and deactivation of the vacuum pumps in order to optimize the consumption of current, for the sensing and measurement stage. All sensors can be activated by means of heating to reach the optimal conditions and obtain a high degree of reliability for the detection of gases. 


\section{Conclusions}

302

303

304

305

306

307

308

309

310

311

312

313

314

315

316

317

318

319

320

321

322

323

324

325

326

327

328

329

330

331

332

333

334

335

336

337

338

339

340

341

342

The wireless electronic nose obtained $97 \%$ of discrimination of the gases by means of PCA and LDA processing methods, which it demonstrated the good versatility of the components (i.e., Arduino, Xbee Modules, Sensors, pumps and other) able to perform a real-time system for an on-line gases monitoring with a low cost and low consumption; additionally with the stand-by-mode at times the data is not being transmitted (i.e. Xbee modules) or data are not being acquired (i.e., short times in sensor reset or heating for reliability of the acquisition data). After the tests were realized, it is observed that this type of wireless electronic noses could reach a high acceptance in different areas of the industrial sector such as: Quality control of raw materials, products processed (to estimate the time of life of a product), classification of essences and perfumes, cosmetic sector, detection of pathogenic microorganisms, etc.

\section{Acknowledgments}

We would like to give our most sincere thanks to the Pamplona University and btB-test Project (H2020 MSCA-RISE-2017 Program - Marie Skłodowsk a-Curie Research and Innovation Staff Exchange (RISE), R\&D under contract no. 777832) by funding an important part of this project.

Author Contributions: Pablo Gómez performed the artificial smell system, writes the manuscript and made the different tests with the system. Cristhian Durán writes the manuscript, provides the technical feedback, and Rafael Acosta revises and proofread the manuscript.

Conflicts of Interest: The authors declare no conflict of interest.

\section{References}

[1] Gardner J., Barlett P. Electronic Noses: Principles and Applications. Oxford University Press, 1999.

[2] Lozano J., Arroyo T., Santos J., Cabellos J. Electronic Nose for wine ageing detection. Sensors and Actuators B: Chemical 133, no1, pp. 180-186, 2008.

[3] Zhang H., Wang J., Sheng Y. Evaluation of peach quality indices using an electronic nose by MLR, QPST and BP network. Sensors and Actuators B: Chemical 134, nº1, pp. 332-338, 2008.

[4] Zhang H., Wang J., Sheng Y. Prediction of acidity, soluble solids and firmness of pear using electronic nose technique. Journal of Food Engineering 86, n³, pp. 370-378, 2008.

[5] Sinesio F., Natale C., Quaglia G., DÁmico A. Use Electronic nose and trained sensory panel in the evaluation of tomato quality. Journal of the Science of Food and Agriculture, nº80, pp. 63-71, 2000.

[6] Durán, C. M. A. (2005). Diseño y optimización de los subsistemas de un sistema de olfato electrónico para aplicaciones agroalimentarias e industria. Universitat Rovira I Virgili.

[7] Quicazán, M., Díaz, A., \& Zuluaga, C. (2011). La Nariz Electrónica, Una Novedosa Herramienta Para El Control De Procesos Y Calidad En La Industria Agroalimentaria. Vitae, 18(45), 209-217.

[8] [11] Panigrahi, S., Balasubramanian, S., Gu, H., Logue, C. M., \& Marchello, M. (2006). Design and development of a metal oxide based electronic nose for spoilage classification of beef. Sensors and Actuators B: Chemical, 119(1), 2-14. http://doi.org/10.1016/j.snb.2005.03.120

[9] Bhattacharjee, S., Roy, P., Ghosh, S., Misra, S., \& Obaidat, M. S. (2012). The Journal of Systems and Software Wireless sensor network-based fire detection, alarming, monitoring and prevention system 
343 for Bord-and-Pillar coal mines. The Journal of Systems \& Software, 85(3), 571-581. 344 http://doi.org/10.1016/j.jss.2011.09.015

345 [10] Longkang, W., Baisheng, N., Ruming, Z., Shengrui, Z., \& Hailong, L. (2011). Zigbee-Based 346 Positioning System For Coal Miners. Procedia Engineering, 26, 2406-2414. 347 http://doi.org/10.1016/j.proeng.2011.11.2452

348 [11] Gualdrón O. (2011). Sistema de olfato electrónico de bajo costo para la detección de diferentes 349 compuestos químicos contaminantes, 121-126.

350 [12] Restrepo Echeverri, D., Ríos Cano, S. H., \& Jiménez Builes, J. A. (2012). Detección y control de 351 atmosferas explosivas en minas subterráneas de carbón usando programación estructurada. Revista

352 de Educación En Ingeniería, 7, 10-21. Retrieved from

353 http://www.educacioneningenieria.org/index.php/edi/article/view/240/

354 [13] Fakundu B., Andrews G., Phylaktou H. (2014). Gas Explosion Venting: Comparison of Square 355 and Circular Vents, Chem Engi Tran, 36 163-8

356 [14] Schuo, X., Xueye, W. E. I., \& Yu, W. (2010). Science and a multipath routing protocol for wireless 357 sensor network for mine security monitoring, 20 (1),148-151. http://doi.org/10.1016/S1674$358 \quad$ 5264(09)60177-0

359 [15] Tiantian, J., \& Zhanyong, Y. (2011). Procedia Engineering Research on Mine Safety Monitoring 360 System Based On WSN, 00, 2146-2151.http://doi.org/10.1016/j.proeng.2011.11.2418

361 [16] Wen, D., Yue, X., Ma, H., \& Wang, Y. (2011). Design of Coal Mine Gas Monitoring System Based 362 On WSN, 00, 2146-2151.http://doi.org/10.1016/j.proeng.2011.11.2418

363 [17] Yuan, Y., Shen, Z., Quan-fu, W., \& Pei, S. (2009). Procedia Earth and Planetary ScienceLong 364 distance wireless sensor networks applied in coal mine. PROEPS, 1(1), 1461-1467.

365 http://doi.org/10.1016/j.proeps.2009.09.225

366 [18] Chen, Set al. (2012). Analysis of the Power Consumption for Wireless Sensor Network Node 367 Based on Zigbee, Procedia Engineering, Vol. 29, 2012, pp. 1994-1998.

368 [19] Geiger, R. (1974). The Anzin Coal Company,

369 https://books.google.com.co/books?id=EOKSwlb81BAC\&printsec=frontcover\&dq=inauthor:\%22Ree

$370 \mathrm{~d}+\mathrm{G} .+$ Geiger\%22\&hl=es-

$371419 \& s a=X \& v e d=0 a h U K E w j F 15 T o 37 T d A h W P w F M K H Y 5 t D J A Q 6 A E I N j A C \# \mathrm{v}=$ onepage\&q\&f=false

372 [20] The eNose Company ${ }^{\odot} 2018$. Available online: http://www.enose.nl/rd/technology/ (accessed on 3732 March 2018).

374 [21] Parker Hannifin Corporation $2018^{\circ}$. Available online:

375 http://www.parker.com/Literature/Precision\%20Fluidics/Miniature\%20Diaphragm\%20Pumps/T2-

376 03\%20Data\%20Sheet.pdf (accessed on 11 September 2018).

377 [22] Alphasense Company ${ }^{\odot}$ 2018. Available online: http://www.alphasense.com/WEB1213/wp-

378 content/uploads/2017/07/MMO-CO.pdf (accessed on 1 January 2018).

379 [23] FIGARO Engineering Inc. Available online:

380 http://www.figaro.co.jp/en/technicalinfo/principle/mos-type.html (accessed on 3 January 2018).

381 [24] Di Benedetto, L. T., Alexander, P. W., \& Brynn Hibbert, D. (1996). Portable battery-powered 382 flow injection analyser for volatile alcohols using semiconductor gas sensors. Analytica Chimica 383 Acta, 321(1), 61-67. http://doi.org/10.1016/0003-2670(95)00564-1 
384 [25] Circuits Today Company ${ }^{\oplus}$. Available online: http://www.circuitstoday.com/lpg-sensor-using385 arduino (Accessed on 4 September 2018).

386 [26] Arduino, T. (2015). Arduino UNO. Retrieved November 5, 2015, from 387 https://www.arduino.cc/en/Main/ArduinoBoardUno

388 [27] Kutop Company.

389 Available online:

390 https://kutop.com/meanwell-ip65-level-clg-150-single-output-switching-power-supply-led-

391 driver.html (Accessed on 4 September 2018).

392 [28] Mohammad, S. (2014). Implementación y Evaluación del protocolo de sincronización RBS para 393 las redes inalámbricas de sensores.

394 [29] TablePlast (2006). IRAM Certification of Manufacturing Conformity and the Safety Mark 395 established by Resolutions S.I.C. \& M. http://www.tableplast.com/sites/default/files/certgpp_1.pdf 